\title{
Improving from Our Current Practices in Education In the Presence of Inputs from Multiple Disciplines
}

\author{
Chandana Watagodakumbura \\ RMIT University, Melbourne, Australia
}

\begin{abstract}
In this paper we discuss some of the limitations in our common practices as educational practitioners. We may not address the needs of a neurodiverse society. Instead, we may incorrectly assume idealistic individuals to exist. There are a number of areas related to assessment that we can improve on following some reflections. We may fail to thoroughly understand the concepts of higher-order learning and put them into practice. The time factor in learning processes may not get the significance it deserves leading to sub-optimal environments. The need to address learner motivation levels may get neglected without understanding the serious consequences. We may allow non-educational bodies to dictate terms on our curricular, sacrificing important pedagogical and human principles. The socio-economic conditions may unnecessarily impact learners much more than individual neurological and psychological characteristics. We present these limitations and suggest how an authentic learning experience can be achieved by overcoming them.
\end{abstract}

\section{Introduction}

Authentic education is identified in this paper mainly as means in which learners' individual psychological and neurological needs are catered. It provides a deeper perspective to education than what we have currently perceived in general. In this paper, the author highlights some ways in which we can provide an authentic learning experience to our learners, minimising some subtly understood negative practices. From section 2 through to 9, we present some of these less-optimal practices we may carry out inadvertently in our teaching-learning environments. Then we suggest possible improvements that lead to truly authentic educational experience, by engaging in reflective practices, and conclude in section 10

\section{The need to address unique psychological and neurological needs}

We have seen a trend in the recent past that educational organisations promote inclusive practices to be applied to teaching-learning environments. At the same time, psychologists who study the characteristics of gifted personnel, promote special educational programs and learning environments for gifted learners [1-2]. As revealed in the studies with enough evidence, gifted personnel show overexcitabilities, namely in the areas of emotional, intellectual, imaginational, psychomotor and sensual [3-5]. These overexcitable characteristics make them react differently, or in unusual manner, to stimuli, or social situations than that would happen or expect normally. For these individuals, over excitable characteristics and behaviour are innate and natural to them and usually last throughout the life span. In our current practices, we tend to accept behaviours without over excitable characteristics as the normal, or the expected average behaviour. We as a society, tend to see those over excitable characteristics and behaviour negatively, possibly as abnormal, despite the fact that individuals possessing these characteristics have the potential to be grate creators, innovators, leaders and scientists, to name a few, if fostered appropriately. The trend in the recent past promoting inclusive practices has not gone much far; the current superficial level of these practices need to go beyond this level to enhance broader understanding and acceptance. The author does not believe that disintegrating the gifted personal from the main education system is a viable solution; rather it should be including them in the main stream provided that inclusive practices are broadly and deeply understood and practiced. In an authentic educational framework[6-7], one of the most important features is to raise the awareness of neurological and psychological diversity within the human species. We are made aware of each other's differences and we become more tolerant and accepting. It is quite natural that we tend to see clones of ourselves in the people we meet and disregard highly possible psychological and neurological difference in two different individuals. In an authentic education framework we attempt address this limitation directly and squarely. We inquire, especially, about possible emotional, intellectual and imaginational overexcitable characteristics of some learners that impact their learning process, and we accept that we need to make allowances for them. Individuals possessing these over excitable characteristics tend to be curious, inquiring, creative and open-minded and yearning for fulfillment of these instincts. In other words, how can we engage in our teaching-learning process so that such individuals are not disadvantaged and identified correctly? How can we construct curricular to meet the needs of these personnel with over excitable characteristics? The author suggested, in an authentic education 
framework, learner evaluations are to be done using generic attributes, rather than the currently used subject area specific grade or numeric mark. The positives of the former is that it is associated with the intrinsic learner characteristics and valid possibly throughout his or her life span; on the other hand, the latter has an extrinsic nature and may be valid for a shorter period of time after the conduction of the assessment. If we can identify an individual with intellectual, imaginational and emotional over excitable characteristics, and are aware of his or her potential, the probable employers can match his or her intrinsic abilities and skills to an appropriate employment category. In this situation, the individual will be at ease, or in equilibrium, with his internal psychological and neurological characteristics and the employer benefiting from a highly functioning, well-tuned employee.

\section{Not to expect the presence of idealistic learners in the learning environments}

The characteristics of learners can be broadly categorised into two, either visual spatial abilities or auditory sequential abilities, as we have discussed so far. When we differentiate between the two categories, it is important to understand that when one possess stronger characteristics in one type, he or she may distant himself or herself from the other type. For example, if one possesses much stronger visual spatial characteristics, he or she may show weaker competencies in auditory sequential skills. However, in learning environments, we tend to expect idealistic individuals possessing strong characteristics of both visual spatial as well as auditory sequential. Even though, it is not impossible to have individuals with well-developed skills in complete spectrum of the auditory sequential and visual spatial continuum, the author argues that expectations of most of the individuals to have these skills in the full spectrum could be unrealistic. In other words, it is we being idealistic to expect a highly sensitive person with imaginational, intellectual and emotional over excitabilities to be easy going and extroverted; it is highly possible that such a person is an introvert [1]. It is as if we are expecting someone with very good abstract thinking abilities to be good at memorising facts, or rote learning, at the same time; gifted learners with good abstract thinking abilities perform much badly in rote learning environments. It is as if we expect an individual with a strong ability to see the big picture to be equally well capable in paying attention to detail. We may expect one who is able do a talk quite fluently, or smoothly, is also equally capable to write a comprehensive essay on the topic. In a similar vein, we may have premeditated negative expectations as well. For example, we may expect that one who finds it difficult to jot down notes well while listening to a talk not being able to provide a comprehensive and satisfactory answer to a written question. We may expect one who is not able to digest a complex high level concept thoroughly not being able attend to a step by step practical work involving the same complex concept. Further, we may expect one who cannot give a reasonable answer to strictly timed question not being able provide a significantly improved answer if we give a reasonably adequate time. It is also well accepted concept that gifted individuals develop asynchronously, meaning that they do not develop in all skill and ability areas at the same speed; some areas of development may lag behind some other more advanced areas. In an authentic education framework, we tend to differentiate learners possessing a more preferred style, while trying to help on improving in the skills related other style, without disregarding the less preferred one outright. Similarly, in the work type differentiation we made as implementers and integrators, it may be idealistic to identify a person with very strong implementer type characteristics as well as very strong integrator type characteristics. It is highly possible that one who is a good implementer is not an equally good integrator, and vice versa.

\section{Issues related to assessment}

Current practices of education leads to a number of less optimal assessment related issues. Learners are usually directed by the assessment in how they engage in the process of learning. If the assessment is conducted in order to test ability to merely recall facts, the learners would usually try to cram the facts in to their heads the night before the assessment or examination. This crammed information will be retained in memory at most a few days, not more, and as a result, it becomes a futile effort in terms of effective learning. If the assessment leads to testing deeper understanding of the content covered, then the learners would have no option but to study and understand the content spending more time throughout the period, possibly a semester, or term. In other words, we highlight here that the need for reflective observation and abstract conceptualisation stages of the Kolb's experiential learning cycle to be significantly focused when setting up assessments; these are the stages in which the learner needs to spend more time, integrating pieces of information and identifying new relationships. This is not to disregard the fact that, knowing mere facts would be very useful in many but simplistic real life day to day situations. For example, one may need to remember a few street names through which he or she may travel towards a new destination; a list of few items may be memorised when going to a shop; one may memorise the names of few people he or she met at a get-together. But the important point is, since facts 
can be relatively easily memorised if a situation demands, it does not need to be practiced or the main focus in a course of education. With the technological advances in the last few decades, information or facts get to your table very easily and cheaply; what is important is to relate this information to other such information and experiences and make more useful deeper meaning or knowledge. Hundreds of years ago, when mere gathering of facts was difficult and expensive, it would have been a great achievement to do so. Despite years of social and technological changes, we still have roots of assessment focusing recall of facts in a number of learning environments. We probably are giving in to years of traditional practices rather than being reflective on our practices. Rote memorisation is identified to be a skill mostly of the left hemispheric brain and as a result, learners with auditory sequential preference will have an undue advantage over visual spatial learners in achievement, but not necessarily in learning. Assessments focusing rote memorisation, as result, are not fair, and alienate visual spatial learners, most of whom also demonstrates gifted characteristics, further. Further, when assessments are focused on rote memorisation, front and back cortices of human brain will be underutilised resulting in an incomplete, or ineffective, learning experience.

Another important aspect of assessment is the time factor. One of the predominant approaches to assessment is to conduct a timed examination at the end of the learning period. Due to the practical difficulties of conducting assessment under examination conditions and other possible reasons such as testing how speedy learners can become in providing answers, we usually have a limited time set for assessments. The dominant negative aspect in lieu of this is that we tend to overload all assessment material into the scarcely available time period. Having covered a vast area of material, we may urge to use as many questions as possible as part of the assessment, within a limited period of time available logistically. We tend to expect the learners to be robots rather than thinking human beings. We incorrectly assume that the higher the speed of providing answers, the higher the ability of the learner; we have wealth of neuroscience findings to contradict this view. Expecting learners to provide premeditated rapid answers in all situations cannot be adequately justified. Human brain is a parallel processor, not a serial or sequential processor similar to machines. Parallel processors consume relatively more time for their operations compared to serial processors, or machines. As we highlighted earlier, reflective observation and abstract conceptualisation stages of the Kolb's learning cycle take more time than merely sensing and memorising; thus we need to allocate learners adequate time to reflect and conceptualise on the answers they provide. We get to know and feel the concept of time using our left hemispheric brain [1] and as a result, we put auditory sequential learners in a more advantages positions, ahead of the visual spatial learners. At least, in setting a time for a timed assessment, we need to allocate enough time for human brains to produce well contemplated answers. If the assessment focuses on facts, then we can argue that memorised facts can be reproduced on the paper relatively quickly mimicking a serial routine processor operation. But for an assessment focusing deeper understanding, it is important that we allocate reasonable amount of time for construction as well as writing down of the answer. Considering all these limitations in timed assessment, it is always a good idea to include nontimed assessment component as part of learner evaluation within a single course or unit. Especially when an assessment component is testing a specific knowledge area or application, we can allow learners to refer to as much material as available for them to come up with a good solution, without having a time restriction. Timed tests can generally and ideally be used to test basic understanding of some concepts, or generalised knowledge, rather than a particular application for which solutions can be provided in premeditated manner, rather than in an open-minded manner. High level concepts, or generalised knowledge, usually require a longer time to be internalised, and once done so, remain in memory longer. Consequently, these high level concepts, or generalised knowledge, usually cannot be crammed in to short term memory in the last minute to mimic effective learning has been accomplished. Further, as highlighted earlier, we have evidence that visual spatial type learners tend to do badly on timed assessment despite possessing good abstract thinking skills; it could be their open-minded and perfectionist approaches, or thinking in pictures approaches, that hamper them down. Thus, carefully managing the time allocated for a test or examination could at least reduce the negative impacts on some learner category such as visual spatial learners, a group that comprises a large portion of gifted learners.

Multiple choice questions are in the common armoury of contemporary educators as a tool used assessing learners. The main advantages of using multiple choice questions are that they can cover a wider area in the content as answering a single question relatively takes less time and are less time consuming to mark, usually using electronic scanners. One of the major drawbacks of this technique is that, especially if not constructed properly, these questions tend to confuse the learners, or at least some perfectionist learners. That is, the learners can interpret the meaning of some statements in a number of different ways, yielding to incorrect or unexpected answers. Some statements we write might superficially mean one thing while 
considered deeply, or more critically, may mean another. Such ambiguities can sometimes be very difficult to avoid. An individual who is naturally inclined to consider matters deeply, or in different contexts, such as visual special learners, may find it difficult to come to a conclusion despite spending longer than usual time for answering [1]. Multiple choice questions can usually be set easily if the focus is on recalling facts, with only one correct answer in a straightforward manner while all the others are quite obtrusively incorrect, but as we mentioned, it is a practice we are trying to avoid. Human brains operate differently when exact answers are sought than when a relative judgement, or evaluation, is performed. For the latter type of operations the learners have to be more reflective and use the back integrative cortex more. Being able to be reflective is a good higher order learning activity compared to obtaining an exact answer following some strict rules, or instructions. From another point of view, a limitation of multiple choice questions is that we are trying to be objective in getting a preformatted answer from the learners rather than a unique answer constructed by the learner. Here, we force our learners to be convergent in thinking as opposed to be divergent; there is more analysis rather than synthesis. In constructivist theory of learning, we highlight the fact that learners uniquely manipulate information in the learning process based on their personnel knowledge and experience; that is they construct meaning uniquely during a learning process. In the assessment, if we get the students to provide their unique authentic answers, instead of premeditated or memorised ones, it could result in a better assessment outcome. Further, we are being biased to our auditory sequential learners, especially in terms of achievement resulting from these assessments. The only resolution is that we have to take extra care in formulating multiple choice questions so that they are clear and free from possible ambiguities and concepts and higher-order learning is assessed instead of specific details. That is, even if we use multiple choice questions, the learners need to be directed to be reflective, performing comparisons and evaluations, instead of being forced to follow routine instructions in order to obtain exact answers. This principle needs to be emphasised irrespective of whether the area of study is in a hard science or soft science area; the author stresses on this point because there is a general belief that it is difficult set reflective and subjective type questions in the areas of hard sciences. This may not necessarily be true, if you decide to be more reflective and creative.

The focus of assessment should be to evaluate how much generalised concepts, or high-level knowledge, learners retain for a longer period at the completion of the study period. In the process of generalisation, the learners abstract the commonalities from a set of available specific information or knowledge to create new knowledge, or relationships. Such generalised concepts or knowledge can usually be retained for longer periods and would be more useful in other areas of study as well, or more simply, in day to day life situations. That is, we will be encouraging our learners use the high-level concepts across multiple domains, or be wiser in the way the concepts are used. It would be less ideal if we test the learners for the ability to recall or apply specific or narrow knowledge for a specific application that may have learned during the study period. Learners tend to forget specific or narrow knowledge gained during a study period very soon, probably within days or weeks; they may succeed in recalling and applying knowledge in an assessment just after the study period, but not beyond that. To improve the validity of assessment, our focus should be to test more generalised knowledge, or high level concepts that learners can and will retain longer, possibly for months or years, and that would be useful more generally. In this way, we encourage our learners to engage in learning in such a way that they can relate what they learn to more general situations or applications, as that is the focus of assessment. The learners will be relived from cramming specific information merely for the purpose of getting a higher grade, or achievement, though not accomplishing deep learning. As highlighted earlier, the assessments should force learners to make evaluations, or judgements, and provide creative, personalised answers instead of expecting exact or factual answers. By doing this, we direct our learners to be reflective conceptualisers using integrative parts of the brain, or the cerebral cortex, more.

Sometimes, over a study period of a semester, or term, we introduce a number of many new learning materials or concepts to our learners, spending considerable amount of time, but focus of assessment may be restricted to only a few areas. In other words, assessment is not properly and fairly aligned, or distributed, to the content learned. Learners may have invested time on learning all areas presented, but would find that assessments are focused only on a few narrow areas or sections. We should try to avoid such situations as the evaluation results may not reflect learner understanding accurately. We may also fail to get the attention of the learners on some important sections of the subject, or may inadvertently direct them to learn strategically to purely get through the assessments; the learners will be more interested in identifying clues such as which parts will be tested in assessments, or examinations, rather than concentrating deeply in understanding, or learning, the content. Learners will be extrinsically motivated merely in pursuit of grades, or achievements, when strategic approaches are encouraged; as a result, to motivate learners 
intrinsically on learning, we need to discourage strategic approaches to learning by not providing avenues for it. Basically, we need to align the assessment to learning and the content properly. Ideally, we should construct assessment constituting material or sections in proportion to the time we utilise to introduce and make the learner understood the same. In this way, we tend to introduce the most important sections or concepts in the subject area, spend a reasonable of time on them in making the learner understand and then follow it up with an evaluation in the assessment. This contrasts from setting up the assessment from only the areas, or sections, preferred by the facilitator or the facilitator is more comfortable in setting up questions. By making assessment aligned with the learning and content, we make assessment more consistent and fairer. Some learners with perfectionist attitudes, such as the visual spatial learners, will be disadvantaged if we are not consistent with the assessment material selection in regard to the time we spend on elaborating them; such inconsistencies direct learners to take a strategic learning approach, which is not the usual preference for visual spatial learners.

\section{Not to have non-educational organisations setting the agenda}

In current practices, it is not uncommon that educational institutes or units play a second fiddle to industrial or professional bodies. Industrial or professional bodies tend to impart guidelines lacking multidimensional perspectives; for them the profession or the industry is more prominent than the learner as an individual, or human being. In an authentic education framework [6-7], we give due recognition to the individual as an authentic personality; it is more important for this framework to develop a balanced, authentic personality than to train him or her for a specific industry or profession needs. It is possible that an individual changes his or her industry or profession a few times during the career span. As a result, it is important for an individual to get an opportunity to develop authentically to his or her natural instincts, to a higher potential level; getting trained in relation to the specifics of an industry or profession becomes secondary. In an authentic education framework, learners get to know themselves, their likes and dislikes, strengths and weaknesses better helping them to look for a specific work category, even within a broad industry or profession, to match the requirements. An authentic education framework is more fundamental and root in helping individuals to develop authentically within a society[6-7] than the possible guidelines set by industry or professional bodies with possible vested interests. That is, an authentic education system promotes the development of the person to his or her full potential, good citizenship with the highest priority, delegating the other industry or profession specific skills development to a secondary level. If an industry or professional body employs an individual, it should be up to them to negotiate with the probable employees to urge them to develop and enhance industry or profession specific skills. It is improper and unjust for industry or professional organisations

to expect the learners to have mastered industry or profession specific narrow set of skills as part of their education. It should be the responsibility of the industry or professional organisation to motivate learners to develop additional, more specific skills, weighing in the future prospects of the industry. Unlike the current practice of looking at an extrinsic testimony, they should be looking at the generic attributes highlighting intrinsic characteristics of the probable employee to match the requirements of the position. Further, these specific skills development should not restrict the individuals to one-sided, or one-dimensional, developments as it would limit authentic personal development as a whole human being. The industry and professional bodies need to be aware and understand their social responsibilities to help individuals to develop to their full potential when imparting guidelines for education.

\section{Overcoming the lack of focus in higher order learning}

As highlighted in the assessment issues section, the focus of learning needs to be beyond pure memorisation of facts, or information. Just because we encounter situations in our day to day lives that we can make use of memorisation of facts usefully in a limited sense, we cannot justify it being used as the focus for learning in many educational environments. We see education more broadly, something that helps individuals to reach their authentic, or unique, full potential, maximising the level efficiency of their social operations; that is, education need to support individuals to develop humanistically, in an all-round manner. When we try to understand the meaning of higher-order learning, Bloom's taxonomy $[8,12]$ is a very useful tool to employ digesting it. At the lower end of the spectrum we have remembering and mere comprehension and at the higher end of the spectrum we have synthesis and evaluation; in the middle levels, we have application and analysis of knowledge. Though most of us understand the essence of this classification broadly, there might be significant differences in the way we apply it to learning and assessment. One common understanding would be that we can use the lower end of the spectrum for relatively lower years of education, or learning while the higher end of the spectrum can be used for higher years of learning. Justification for pursuing this approach is that the 
tasks targeting the higher end of the spectrum are usually more difficult and should be deferred to relatively older students. For example, for undergraduate students, we may use a lower level of the classification and for postgraduate students we may use the higher end of the classification. The author argues that this is an incorrect simplification, and for all years, or levels we need to have the focus mainly on the highest end of the spectrum, even though we may make use of the other levels of the full spectrum. That is, at any year, or level, we may have learners who would like to get the comfort at the lower as well as higher level of the spectrum, for various reasons; our duty, as educators, should be to push everyone towards following higher end practices, and thereby for higher order learning. Paying special attention on abstract conceptualisation and reflective observation stages of the Kolb's experiential learning cycle, utilising the integrative parts of the cerebral cortex, is important in this regard. We, as educators, can push our learners to engage mainly in the higher order learning activities by aligning the assessment components towards that end, as learners are mainly guided by the assessment. We have both the auditory sequential learners who usually prefer to attend the tasks targeting the lower end of the spectrum and the visual spatial learners who prefer to attend the tasks targeting the higher end of the spectrum. In other words, left hemisphere of the brain usually performs the functions related to the lower end of the spectrum while the right hemisphere is more inclined to do the tasks in the higher end of the spectrum. As we have highlighted earlier, for ideal learning to take place, we essentially need to get the right hemisphere involved; deep learning cannot be achieved without its involvement. We also have to use the Bloom's taxonomy as a tool for identifying intrinsic learner characteristics, but not just to discriminate one end at the expense of the other. In the section highlighting the balance of practical work and theory in a learning environment, we pointed out the significance in practical work even though on Bloom's spectrum they may fall towards the lower end; in a practical work session, we may mostly follow some instruction to accomplish a task and may not have engagements related to synthesis and evaluation. But we engage in practical work in a learning environment to enhance our understanding on abstract theories. We also pointed out that assessment on practical work may even be of formative type. To reiterate, we have both auditory sequential and visual spatial learners and implementer and integrator type work in our society. We accept their unique contributions to our social operations; the balance of the society is maintained due to the unique operations of all categories. However, in a learning environment, we attempt to give mixed exposures to all learners; that is if an individual is identified to prefer a learning context highlighting lower end of the spectrum, we encourage them to take special measures in practising learning contexts at the higher end of the spectrum; we do this because it will be useful for an individual to develop skills pertaining to both ends of the spectrum, in their social operations.

Another limitation of our current practices is that we tend to, either inadvertently or not, set a lower ceiling of the spectrum to our learning and assessment environment. That is, we may focus on analysis or application as the highest possible level in the teaching-learning practices, disregarding higher levels of synthesis and evaluation. In any arbitrary learner cohort, we may have learners with characteristics preferred to either higher end of the spectrum or lower end of the spectrum; if we set a ceiling at a middle level, we are disregarding some learner category. Put differently, we may not emphasise all four stages, more specifically the abstract conceptualisation stage, of the Kolb's cycle. This is something we are trying to avoid in an authentic education framework; we endeavour to give every learner an opportunity to develop their authentic characteristics, to make them at ease with their natural innate psychological and neurological characteristics. We also have a common inclination to assume that highest levels of the spectrum, namely synthesis and evaluation, can only be applied to research education; in research activities we more specifically talk about creations, or innovations, or synthesis, so to speak. But the author argues that this is really not the only instance we can emphasise synthesis, as we can look for unique, creative answers learners provide especially for open-ended question we use for assessment. To answer openended question, learners have to consider different viewpoints and see relationships among different contexts, or areas; by doing so, they synthesise new knowledge, and what is important is this process of synthesis, or creation. The open-ended answers then have to be validated using other parameters such as accuracy, relevance and logicalness, so on and so forth. Further we can encourage and provide an appropriate environment for learners to develop open-minded thinking in the learning process and to provide authentic, or unique, and creative solutions to possible open ended questions we set. Despite our inclination to accept relative difficulty on conducting courses in hard science areas with a focus of enhancing learner creativity, the author argues we still can achieve that on a properly set and focused curriculum, including assessment.

\section{Overcoming the lack of attention to the time factor in learning}

In our current practices, one of the most common limitations would be not giving enough attention to the time factor, irrespective of whether it is on 
assessment or the whole teaching-learning process. One significant point we need to highlight here is that the human brain or the neural system is a parallel processor. It takes in a large number of inputs and attempts to recognise patterns in a learning process based on feedback cycles to produce the output. This pattern recognition process is usually very complex and takes more time than serial processing in which relatively less number of inputs is taken in, send them through a number of repetitive processing cycles rapidly, before producing the output that is very precise in nature; typical computers or machines operate in the latter manner [10]. We tend to disregard this significant difference in how the human brain operates and how typical computers, or machines, operate in our practices in teaching-learning; in our teaching learning environments we need to provide enough time for human brains to absorb information and engage in a learning process, or constructing meaning, before giving the output. Provision of adequate time is important while delivering information as that happens in a lecture as well as in assessment. For this very reason we highlighted the importance of using a dialectic approach ahead of didactic approach in a teaching-learning environment. We allow and encourage learners to construct meaning in a learning process, considering all the inputs they take in, rather than merely committing to short term memory. We need to provide as many related and useful inputs as possible along with adequate time for them to construct meaning and produce a lasting learning outcome. The more the effective time we deal with any set of information, identifying relationships and different contexts, the longer they would be in our mind, to be recalled when necessary. We can direct human brains to focus on what they are good at doing and get typical computers, or machines, to do what they are capable of, with a clear distinction. There is no benefit in trying to force human brains to emulate machines in the face of process automation tasks that make routine human labour redundant, a common trend observed in the recent past.

In a similar manner, when assessing student learning, we need to provide them enough time to construct the solution based on the given number of inputs. It is not uncommon in our current practices that we assume that the higher the speed at which one can write, or complete, an answer, the higher the capability, or intelligence of the learner; that is, we assume the speed is directly proportional to the ability or intelligence. This fact is reflected even in many contemporary academic, industry or profession oriented standardised testing systems that use

multiple choice questions. They appear to be more concerned about the speed than one's ability to make an accurate and balanced decision based on reflection. Rapidly obtaining a specific, convergent type answer, similar to an output from a machine, or robot, is more important that a reflective, divergent type more elaborate evaluation, or judgement. We are forced to question here that whether the industry, or profession specific, operations require robotic human beings, not thinking well developed individuals; are we forced to sacrifice here broad human development for narrow economic gains, either for us or our organisation? We are stressing here the point that the assessments we mention should essentially not be based on mere recalling of specific facts; recalling facts can probably be done rapidly, if they are committed to short term memory and the nature of the assessment requires such practice. As we pointed out in the assessment issues section, merely testing the learners' ability to recall facts would not constitute a proper assessment, or educational experience. Also, when learners construct their answer, we need to give them enough time to write them down on the paper. If we do not give enough attention to these timing factors when setting up assessment, it is possible that learners perform badly in the assessment not due to their lack of deep learning, but due to the way the assessment is structured. Even worse, some learner categories such as visual spatial learners tend to get more disadvantaged in these circumstances than others; they perform relatively badly on timed tests due to lack of sense about the concept of time, innately. Further, it is highly possible that their speed of hand writing is relatively low compared to the speed of thinking.

We can have another viewpoint on this timing issue in the following manner. Usually, if we keep on practicing the same thing for a longer period of time, we tend to improve on the time taken to complete it through the process of habituation; we can bypass any reflecting and conceptualising stages, speeding up the overall operation. Sometimes these improvements can be seen over many years. If one is employed in an environment that requires him or her to attend to the same routine task for years, he or she may achieve a very high speed in doing that; we assume here that the person overcame the boredom involved by the motivation of having a means of income for the purpose of making the basic needs satisfied. What it suggests is that if one has a valid reason, or purpose, the speed can be achieved relatively easily through repetition. However, in a typical learning environment, learners take up a course for a period of a few weeks. They might be taking a few other similar courses in parallel as well in a programme of study. Consequently, it is not appropriate for us to expect learners to reach a speed that would be demonstrated by one with years of experience. We have to be realistic in the speed, or the response time, in which learners could provide answers after a study of a few weeks. We highlighted this point here because it is not very uncommon that learners comment on insufficient time for completing 
an assessment in many teaching-learning environments. Another common current practice is that learners rehearse repetitively using a large number of previous assessment questions to improve on the speed of answering. If a teaching-learning environment pushes a learner to such a situation, that violates a fundamental principle of learning - speed is more important than reflective and conceptual understanding. Further, research studies show that visual spatial learners dislike repetitive learning activities and as a result such practices make them vulnerable to the circumstances.

\section{Overcoming the lack of focus in learner motivation level}

Sometimes, the performance of the learners is hampered due to lack of learner motivation [13] level. We will have learners who just need to get through a particular course with a pass or merely taking a course because there are no better alternatives; it may be that the particular course is not in their main interest list, or their focused career paths. Even we may not know how many of the learners taking the course would end up following a career path highlighted in the subject area. It is not uncommon that we get to hear from past learners about some of the courses they have undertaken, covering very narrow specific material, have never been used, or being useful, afterwards. Disregarding these facts, we may conduct the course in a narrow way, targeting more specific knowledge areas and applications. In general, we can increase the level of learner motivation by structuring the course with a focus to generalised, or broader, knowledge or concepts. Such generalised knowledge or concepts highlighted in any course would be more useful for learners in a general way, probably in learning other courses or in day to day life situations or personal development. Also, such generalised knowledge or concepts will last longer in the minds, increasing the usefulness. To achieve this, we can relate the subject matter highlighted in the course to more generic applications or situation, rather than the subject specific ones; then we can construct the assessment having this focus in mind as the learners are usually guided by the assessment. If the learners are

convinced that a particular course would possibly help them in a more generic way, irrespective of their career or personal interests, they would be more motivated in investing their time to learn it. Generally, the higher the level of motivation, the more the effective time spend on learning it, possibly yielding better learning outcomes or objectives. Thus, as educators, it is now our responsibility to present the contents in a more generalised manner, making them more useful in a wider range of applications.

\section{Not to have over reliance of education on socio-economic conditions}

Our contemporary education system is entangled much with the socio-economic conditions of the individual families, communities and larger societies. The drawback of this situation is that individual intrinsic learner characteristics get supressed by the more extrinsic measures of socio-economic conditions; while low-socio-economic conditions would typically restrict the opportunities for learners, overly satisfied socio-economic conditions would reduce the motivation level[13] of learners. In such an environment, it is difficult for us to focus on authentic education fulfilling individual learner needs. We tend to provide an education we assume fits everyone equally, disregarding intrinsic individual learner characteristics. Subject to the prevailing socio-economic conditions, we push everyone in the community or larger society along a sole path that is presumed to meet the community or larger societal needs rather than individual learner needs. The learners will not have a preference to choose their direction of learning; instead they will succumb to the pressure from the society to make selections less ideally. These situations are more prevalent in highly competitive societies, with highly negative impacts in terms of sustainable social developments; the typical practices of these environments usually lead to maintain the existing status quo, despite unsustainable, rather than providing means of coming out of the entangle, in the direction of sustainability. Over reliance of education on socio-economic conditions more explicitly have a negative impact on visual spatial, or gifted, learners as their vulnerabilities such as high emotional overexcitabilities get more exposed to less than ideal conditions. It is not uncommon to find in the literature on the gifted how these delicate individuals get destroyed by highly competition oriented societies [3-4, 14-15].

\section{Conclusion}

This paper highlights some common but subtly understood limitations of our general pedagogical practices. The importance of giving careful consideration to them through reflective practices is emphasised. By doing this, we are able to give our learners a more authentic learning experience in a fairer and more just manner.

\section{References}

[1] Silverman, L. K. 2002. Upside-Down Brilliance: The Visual-Spatial Learner, Denver: DeLeon Publishing.

[2] Silverman, L.K. 1998. Personality and Learning Styles of Gifted Children. In Excellence In Educating 
Gifted \& Talented Learners (3rd ed), ed. Van Tassel Baska, Denver, Colorado, USA: Love Publishing Company.

[3] Dabrowski, K. (with Kawczak A. and Piechowski M. M.). 1970. Mental Growth through Positive Disintegration. London: Gryf Publications

[4] Dabrowski, K. 1972. Psychoneuroses Is Not An Illness. London: Gryf Publications

[5] Dabrowski, K. 1977. Theory of Levels of Emotional Development (vol 1) - Multilevelness and Positive Disintegration. New York: Dabor Science Publications.

[6] Watagodakumbura, C. 2013, 'Benefits of authentic education', in Proceedings of the Clute Institute International Academic Conference 2013, Ronald C. Clute (ed.), The Clute Institute, Colorado, USA, pp. 199-203

[7] Watagodakumbura, C. 2012, 'Improving student learning through multidisciplinary perspectives', in Journal of Teaching and Education, International Journal of Arts \& Sciences, Rhode Island, United States, vol. 1, no. 5, pp. 261-267 ISSN: 2165-6266

[8] Biggs, J. 2003. Teaching for Quality Learning at University (2nd ed.). Buckingham, Society for Research into Higher Education and Open University Press.

[9] Ramsden, P. 2003. Learning to Teach in Higher Education(2nd ed.). London: RoutledgeFalmer.

[10] Beale, R. and T. Jackson. 1990. Neural Computing An Introduction. Institute of Physics Publishing

[11] Paul, R. and L. Elder. 2000. Critical Thinking - Tools for Taking Charge of Your Learning and Your Life. Pearson Education.

[12] Entwistle, N. J. 1998. Approaches to Learning and Forms of Understanding. In Teaching and Learning in Higher Education, ed. B. Dart and G. Boulton-Lewis, 72101. Melbourne, Australia: Australian Council for Educational Research

[13] Maslow, A. 1987. Motivation and Personality. Hong Kong: Longman Asia Ltd.

[14] Webb, J. T. 2008. Dabrowski's Theory and Existential Depression in Gifted Children and Adults. Paper presented at the Eighth International Congress of the Institute for Positive Disintegration in Human Development, August 79, Alberta, Canada.

[15] Silverman, L. K. 2004. May. At- Risk Youth and the Creative Process. Paper presented at ARTernatives for AtRisk Youth Conference, May 14, Colorado Springs. 